\title{
Optimal Wire Shape with Consideration of Coupling Capacitance under Elmore Delay Model *
}

\author{
Youxin Gao and D.F. Wong \\ Department of Computer Sciences, University of Texas at Austin, Austin, Texas 78712
}

\begin{abstract}
In this paper, by using calculus of variations, we determine the optimal shape for a wire under the Elmore delay model. Coupling capacitance has been taken into consideration explicitly by treating it as another source of grounded capacitance. Given two wires in parallel, one has uniform width and the other has non-uniform width whose shape is described by a function $f(x)$. Let $T_{D}$ be the delay through the non-uniform wire. We determine $f(x)$ such that $T_{D}$ is minimized. We also extend our study to the case where a non-uniform wire has two neighboring wires. Our study shows that the optimal shape function satisfies an integral equation. Numerical methods are employed to solve the corresponding differential equation and carry out the integration. We provide an efficient algorithm to find the optimal solution. Experiments show that it only takes several iterations to get the optimal results by using our algorithm. Our experiments also show that the wire delay $T_{D}$ is a convex function of the wire width at the driver end.
\end{abstract}

\section{Introduction}

In today's deep sub-micron design, interconnect delay becomes an important factor in determining total delay of a system. To reduce the interconnect delay, wire-sizing is found to be an effective way. To best describe the wire performance, it is believed that wire capacitance should include at least area, fringing and coupling capacitance. [1,7] have found the optimal wire shape to minimize the interconnect delay considering only the area capacitance. $[2,6]$ later on solve the similar problem by taking fringing capacitance into consideration. [8] extends [2,6]'s work to bi-directional wire. Unfortunately, coupling capacitance has not been taken into consideration in these work. Coupling capacitance can be comparable to and even bigger than the sum of area and fringing capacitance as the distance between two adjacent wires decreases $[10,11]$. It plays an important role in determining both the wire delay and the crosstalk effect. To minimize the effect caused by coupling capacitance, one approach is to find the best routing pattern $[12,13]$ to avoid two wires to be placed too close to each other. Another approach is to appropriately size the wire shape to minimize delay and crosstalk effect.

In this paper, we will focus on minimizing the delay effect caused by coupling capacitance. By using calculus of variations, we determine the optimal shape for a wire under the Elmore delay model. Coupling capacitance has been taken into consideration explicitly by treating it as another source of grounded capacitance. Given two wires in parallel, one has uniform width and the other has non-uniform width whose shape is described by a function $f(x)$. Let $T_{D}$ be the delay through the non-uniform wire. We determine $f(x)$ such that $T_{D}$ is minimized. We also extend our study to the case where a non-uniform wire has two neighboring wires. Our study shows that the optimal shape function satisfies an integral equation. Numerical methods are employed to solve the corresponding differential equation and carry out the integration. We provide an efficient algorithm to find the optimal solution.

${ }^{*}$ This work was partially supported by the Texas Advanced Research Program under Grant No. 003658288 and by a grant from the Intel Corporation.
Experiments show that it only takes several iterations to get the optimal results by using our algorithm. Our experiments also show that the wire delay $T_{D}$ is a convex function of the wire width at the driver end.

Due to space limitation, we only show our main results as lemmas and omit the proofs to these lemmas.

\section{Elmore Delay Model}

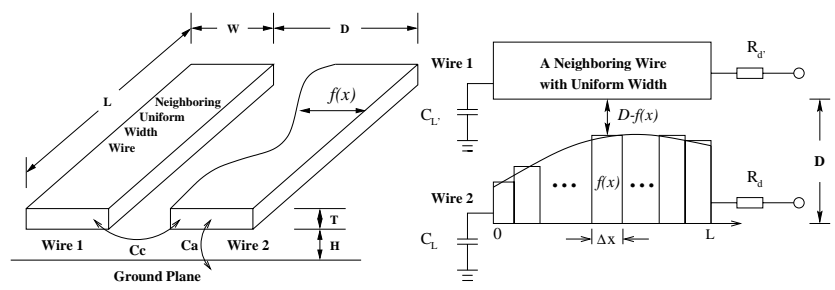

(a)

(b)

Figure 1. Coupling capacitance between two adjacent wires.

For two adjacent wires shown in Fig.1, wire 1 has a uniform width $W$, and wire 2 has a non-uniform width which is described by a function $f(x)$. We partition wire 2 into $n$ equal-length wire segments, each of length $\Delta x=\frac{L}{n}$, and approximate it as a distributed RC network. Let $x_{i}=i \Delta x, 1 \leq x \leq n$. For segment $i$, there is a coupling capacitance between $i$ th segment and neighboring wire. The value is inversely proportional to the distance $D-f\left(x_{i}\right)$, i.e. $C_{c, i}=\frac{c_{c} \Delta x}{D-f\left(x_{i}\right)}$ where $c_{c}$ is unit coupling capacitance.

It has already been found that $[10,11]$, if two adjacent wires are driven by "in-phase signals", i.e. two driving signals are going from low to high at the same time, the coupling capacitance does not play a role in determining either delay or crosstalk effect. If these two wires are driven by "off-phase signals", i.e. one signal is going form low to high and the other is going from high to low, the role of coupling capacitance is the same as that of a distributed wire with an extra grounded capacitance $2 C_{c}$. Since in practice it is not easy to determine whether two driven signals are "in-phase" or "off-phase", for safety we can assume the worst case in our study. We always take the coupling capacitance into delay consideration and treat it as another source of grounded capacitance added to the original distributed $R C$ network. Therefore, the capacitance and resistance of segment $i$ can be approximated by $C_{i}=\left(C_{a, i}+C_{c, i}\right)=\left(c_{0} f\left(x_{i}\right)+c_{f}+\frac{c_{c}}{D-f\left(x_{i}\right)}\right) \Delta x$ and $R_{i}=r_{0} \Delta x / f\left(x_{i}\right)$, respectively, where $c_{0}$ is unit area capacitance, $c_{f}$ is unit length fringing capacitance, and $r_{0}$ is unit resistance. Let $T_{D}$ be the Elmore delay through wire 2 . The commonly used Elmore delay of the distributed RC network is given as the sum over all segment resistance $\left(R_{i}\right)$ multiplied by its down stream capacitance $[4,9]$. In this paper, because of the similar reason explained in [8], we use an alternative but equivalent form of Elmore delay where $T_{D}$ is given as the sum over all segment capacitance 
multiplied by its upstream resistance, i.e.

$$
\begin{aligned}
T_{D} & =\sum_{i=1}^{n}\left(c_{0} f\left(x_{i}\right)+c_{f}+\frac{c_{c}}{D-f\left(x_{i}\right)}\right) \Delta x \\
& \times\left(R_{D}+\sum_{j=i}^{n} \frac{r_{0} \Delta x}{f\left(x_{j}\right)}\right)+C_{l}\left(R_{D}+\sum_{i=1}^{n} \frac{r_{0} \Delta x}{f\left(x_{i}\right)}\right)
\end{aligned}
$$

As $n \rightarrow \infty, T_{D}$ thus becomes

$$
\begin{aligned}
T_{D} & =\int_{0}^{L}\left(c_{0} f(x)+c_{f}+\frac{c_{c}}{D-f(x)}\right) \\
& \times\left(R_{d}+\int_{x}^{L} \frac{r_{0} d t}{f(t)}\right) d x+C_{l}\left(R_{d}+\int_{0}^{L} \frac{r_{0} d x}{f(x)}\right)
\end{aligned}
$$

\section{Shaping a Wire with One Neighboring Wire}

Lemma 1 To minimize the delay in (1), the optimal shape function $f(x)$ satisfies the following first order integral equation:

$$
\frac{c_{c} D}{(D-f(x))^{2}}+2 c_{0} f(x)=\frac{c-c_{f} r_{0} u(x)}{R_{d}+r_{0} u(x)}
$$

where $u(x)=\int_{x}^{L} \frac{d x}{f(x)}$, and c is a constant.

(2) is derived by using calculus of variations [5]. Similar derivations can be found in $[7,8]$. Unfortunately, it is difficult to get an analytic solution to (2). This is different from previous work in $[7,8]$. In $[7,8]$, the Euler's differential equations which are similar to (2) are derived, and they can be solved analytically. In [7], the solution is an exponential function; whereas in [8], the solution is expressed in terms of Lambert's $W$ function. In this paper, we use numerical method to solve (2). is

Let $x=L$ in (2), since $\left.u(x)\right|_{x=L}=0$, a direct result from (2)

$$
c=R_{d}\left(2 c_{0} w_{0}+\frac{c_{c} D}{\left(D-w_{0}\right)^{2}}\right)
$$

where we denote $w_{0}=\left.f(x)\right|_{x=L}$ as the wire width at driver end. The importance of (3) is that given $c$ or $w_{0}$, we can easily calculate the other. In addition, $c$ is a convex and monotonically increasing function of $w_{0}$.

Obviously, the solution $u(x)$ (or $f(x)$ ) only depends on one constant $c$. Substitute (2) into delay expression in (1), we get

$$
\begin{aligned}
T_{D} & =\int_{0}^{L}\left(\frac{C_{l} r_{0}}{f(x)}+\frac{c_{f}+c_{0} f(x)+\frac{c_{c}}{D-f(x)}}{c_{f}+2 c_{0} f(x)+\frac{c_{c}}{(D-f(x))^{2}}}\right. \\
& \left.\times\left(c+R_{d} c_{f}\right)\right) d x+R_{d} C_{l}
\end{aligned}
$$

Without solving equation (2), the following property of function $f(x)$ still holds.

Lemma 2 The optimal shape function $f(x)$ satisfying (2) is a monotonically increasing function.

Let $A=\frac{c-c_{f} r_{0} u(x)}{R_{d}+r_{0} u(x)}$, we can easily derive the following equation from (2).

$$
u^{\prime}(x)=v^{\frac{1}{3}}+\frac{1}{9} w+\frac{2}{3} \frac{c_{0} h+A}{c_{c}-A h}
$$

where

$$
\begin{aligned}
v & =\frac{1}{27} \frac{9 c_{c} M^{2}+27 c_{0} c_{c}\left(D A+c_{c}\right)-D M^{3}}{D N^{3}} \\
& +\frac{\sqrt{3}}{9} \frac{\left(27 D c_{0}^{2} c_{c}-M^{3}\right)^{\frac{1}{2}}\left(c_{c} D\right)^{\frac{1}{2}}}{D N^{2}} \\
w & =\frac{3 c_{c}\left(4 D c_{0}+A\right)+D M^{2}}{D N^{2} v^{\frac{1}{3}}} \\
M & =A-2 c_{0} D \\
N & =c_{c}-A D
\end{aligned}
$$

(5) is a first order ordinary differential equation of $u(x)$. As long as we know $c$, starting with the boundary condition $u(L)=0$, $u(x)$ (and equivalently $f(x)$ ) can be solved numerically by using Runge-Kutta method or other multi-step methods. Then $T_{D}$ can be calculated through (4) numerically. In previous work [7], it is shown that $T_{D}$ is a convex function of $c$, thus any local minimum value for $T_{D}$ is also the global minimum. Later on in our experiments, it is observed that numerically calculated $T_{D}$ is still convex in $c$.

If the driver end width $w_{0}$ is given, we can solve $f(x)$ from (5) directly. Furthermore, given a range of the drive end width, we can search for the optimal solution that can minimize $T_{D}$ within the range.

To find the optimal $c$ which minimizes $T_{D}$ in general, we propose an algorithm based on the following fitting formula of $T_{D}$,

$$
T_{D}=\frac{\alpha}{c}+\beta c
$$

where $T_{D}$ is defined as a function of $c$, and $\alpha$ and $\beta$ are undetermined coefficients. Given two points $\left(c_{1}, T_{D 1}\right)$ and $\left(c_{2}, T_{D 2}\right)$, we thus can solve $\alpha$ and $\beta$. (6) has a unique minimum value at point

$$
c=\sqrt{\frac{T_{D 2} c_{1}-T_{D 1} c_{2}}{T_{D 1} c_{1}-T_{D 2} c_{2}}} \sqrt{c_{1} c_{2}}
$$

The idea of choosing such a special kind of formula originates from previous work [7] on solving the optimal shape function without considering fringing capacitance. In [7], $T_{D}$ is calculated as

$$
T_{D}=R_{d} C_{l} e^{2 r_{0} c_{0} L / c}+\frac{1}{2} c L
$$

which is obviously convex in $c$. But adopting this as a fitting formula will be very expensive to solve for coefficients like $\alpha$ and $\beta$. Probably, it will rely on solving a system of nonlinear equations. (6) can be thought of as a first order Taylor series expansion of (7)

Our algorithm starts with the solution without considering the coupling capacitance, where it provides us with an initial value of $c$. The driver end width $w_{0}$ is thus $w_{0}=c /\left(2 R_{d} c_{0}\right)$ by letting $c_{c}=0$ in (3). The algorithm in the following then searchs for the optimal $c$ which minimizes $T_{D}$.

Algorithm Find the optimal $c$ to minimize $T_{D}$

1. $w_{1}=w_{0}, c_{1}=R_{d}\left(2 c_{0} w_{1}+\frac{c_{c} D}{\left(D-w_{1}\right)^{2}}\right)$

2. $w_{2}=0.8 w_{0}, c_{2}=R_{d}\left(2 c_{0} w_{2}+\frac{c_{c} D}{\left(D-w_{2}\right)^{2}}\right)$

3. calculate $T_{D 1}\left(c_{1}\right), T_{D 2}\left(c_{2}\right)$

4. $c=\sqrt{T_{D 2} c_{1}-T_{D 1} c_{2}} \sqrt{c_{1} c_{2}} / \sqrt{T_{D 1} c_{1}-T_{D 2} c_{2}}$

5. calculate $T_{D}(c)$

6. choose two smallest in $\left\{T_{D 1}, T_{D 2}, T_{D}\right\}$ as new $T_{D 1}, T_{D 2}$

7. choose the corresponding $c$ 's as $c_{1}, c_{2}$

8. repeat 4-6 until no improvement on successive $T_{D}$ 's

"Calculate $T_{D}(c)$ " in the above involves implicitly two steps: first given $c$ solve $u(x)$, and then carry out the integration for $T_{D}$ numerically. 


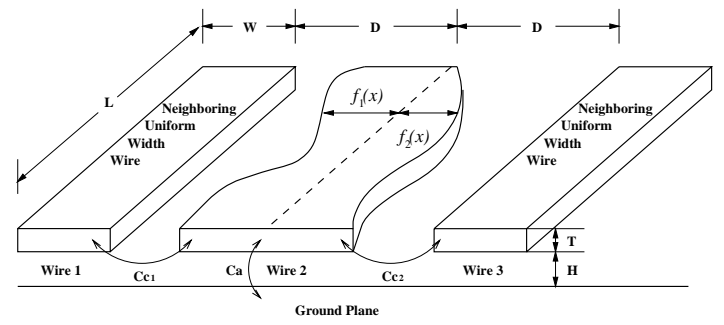

Figure 2. Coupling capacitance between three adjacent wires. The distance between wire 1 and wire 3 is $2 D$.

\section{Shaping a Wire with Two Neighboring Wires}

In this section, given three adjacent wires shown in Fig.2, we will shape wire 2 so that the delay through it is minimized. Wire 1 and 3 have uniform widths. For wire 2, there are two sources of coupling capacitance, one is between wire 1 and wire 2 , and the other is between wire 2 and wire 3. In Fig.2, we define the centerline of spacing between wire 1 and wire 3 as the reference line. The shape of wire 2 is defined by two functions $f_{1}(x)$ and $f_{2}(x)$ with respect to the reference line. If the insulators among these wires are the same, we have the following lemma.

Lemma 3 The optimal wire shape to minimize the delay through wire 2 will have $f_{1}(x)=f_{2}(x)$, i.e. the shape is symmetric with respect to the center-line of spacing.

A direct result from Lemma 3 is that the study in section 3 which is dealing with one neighboring wire can also be extended to shaping the wire with two neighboring wires. The shape of wire 2 can be defined by a single function $f(x)$, therefore the delay is calculated by

$$
\begin{aligned}
T_{D} & =\int_{0}^{L}\left(2 c_{0} f(x)+\frac{2 c_{c}}{D-f(x)}+c_{f}\right) \\
& \times\left(R_{d}+\int_{x}^{L} \frac{r_{0} d t}{2 f(t)}\right) d x+C_{l}\left(R_{d}+\int_{0}^{L} \frac{r_{0} d x}{2 f(x)}\right)
\end{aligned}
$$

where "2" occurs because of two neighboring wires. Comparing with equation (1), the only difference is that we have different parameters (e.g. $c_{0}$ changes to $2 c_{0}$ ). Solving for $f(x)$ can follow the same procedure in section 3 .

For a more general case where the insulators surrounding wire 2 are different, we have to solve for two functions $f_{1}(x)$ and $f_{2}(x)$ simultaneously. We thus have two first order differential equations like (2) to solve. Solving $f_{1}(x)$ and $f_{2}(x)$ can be done iteratively by assuming one has fixed value and solving for the other.

\section{Extension to Constrained Wire-sizing}

Our study can be extended to constrained wire-sizing. In constrained wire-sizing, we are given upper bound $W_{\max }$ and lower bound $W_{\min }$. It is required that $W_{\min } \leq f(x) \leq W_{\max }$, $0 \leq x \leq L$. In this section, we only present the extension of shaping a wire with one neighboring wire. A similar study can be extended to shaping a wire with two neighboring wires. Obviously, if the optimal wire shape function obtained for the unconstrained case lies within bounds $\left[W_{\min }, W_{\max }\right]$, then $f(x)$ is also optimal for constrained wire-sizing. On the other hand, if for some $x, f(x)$ is not within $\left[W_{\min }, W_{\max }\right]$, we find that the optimal constrained wire shape function can be any one of the 5 different types of functions $[1,2]$, i.e. type-ABC, type-AB, type-BC, type-A and type-C, which are shown in Fig.3. These types have at most three parts. Part-A and part-C represent uniform width parts having widths $W_{\min }$ and $W_{\max }$ respectively. Part-B represents non-uniform width part which is described by a function $f(x)$. To determine which type is optimal, we calculate the minimum delay each type can achieve. We then have five delay values from five types respectively. The type which gives the minimum delay value is thus the optimal shape.

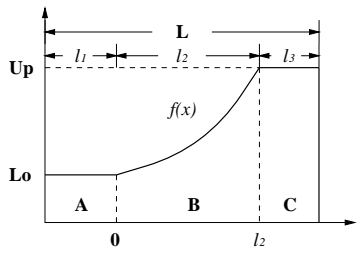

(a) Type-ABC

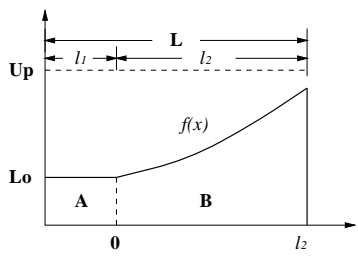

(c) Type-AB

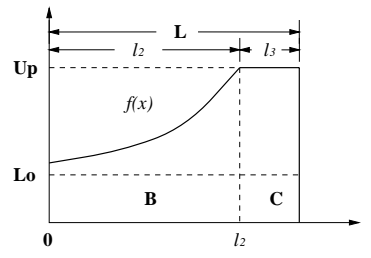

(b) Type-BC

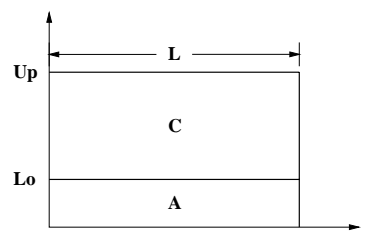

(d) Type-A \& Type-C
Figure 3. 5 different types of optimal shape functions under constraint: Type-ABC, Type-BC, Type-AB, Type-A and Type-C.

For illustration, we show briefly how to calculate delay for type$\mathrm{ABC}$. The total delay through part-A, part-B and part-C can be calculated as:

$$
\begin{aligned}
T_{D} & =\left(C_{l}+W_{\min } l_{1} c_{0}+l_{1} c_{f}+\frac{c_{c} l_{1}}{D-W_{\min }}\right) \\
& \times\left(R_{d}+\int_{0}^{l_{2}} \frac{r_{0} d x}{f(x)}+\frac{r_{0} l_{1}}{W_{\min }}+\frac{r_{0} l_{3}}{W_{\max }}\right) \\
& +\int_{0}^{l_{2}}\left(c_{0} f(x)+\frac{c_{c}}{D-f(x)}+c_{f}\right)\left(R_{d}\right. \\
& \left.+\int_{x}^{l_{2}} \frac{r_{0} d t}{f(t)}+\frac{r_{0} l_{3}}{W_{\max }}\right) d x+\left(R_{d}+\frac{r_{0} l_{3}}{W_{\max }}\right) \\
& \times\left(W_{\max } l_{3} c_{0}+l_{3} c_{f}+\frac{c_{c} l_{3}}{D-W_{\max }}\right)
\end{aligned}
$$

We find that $f(x)$ still satisfies equation (2) in order to minimize delay, except that $R_{d}$ is replaced by $R_{d}+\frac{l_{3} r_{0}}{W_{\max }}$. The shape function $f(x)$ for part-B can be determined by using the algorithm we introduced. Since its driver end width is $W_{\max }$, constant $c$ in (3) can be calculated directly. Solving equation (5) thus gives us $f(x)$. On the other hand, the load end width is fixed at $W_{\min }$, so the wire length $l_{2}$ is determined. The total delay $T_{D}$ is then expressed as a function only depends on $l_{1}$ and $l_{3}$, since $l_{1}+l_{2}+l_{3}=L$. To determine $l_{1}$ and $l_{3}$, we solve $\partial T_{D} / \partial l_{1}=0$ and $\partial T_{D} / \partial l_{3}=0$ simultaneously. Delay calculations for other types can follow the similar procedure.

\section{Experimental Results}

In this section, we show some experimental results of shaping a wire with one neighboring wire. We use Runge-Kutta method to solve the ordinary differential equation (5). Then we apply Simpson's rule to carry out the integration for calculating $T_{D}$ in (4). The circuit parameters are chosen as follows: $L=3,000 \mu \mathrm{m}, r_{0}=$ $0.03 \Omega / \square, c_{0}=0.2 \mathrm{fF} / \mu \mathrm{m}^{2}, c_{f}=0.2 \mathrm{fF} / \mu \mathrm{m}, C_{l}=10 \mathrm{pF}$. Other parameters, such as $R_{d}, c_{c}$ and $D$ are subject to change in different case studies. Those parameters are listed in Table 1. In addition, we also list the driver end width $w_{0}$ for each case without considering coupling capacitance, since we can solve them in advance. Those widhts are the initial values when we use our algorithm to find the optimal shapes. The formulas involved in this step of calculation can be found in [8].

Case 1 and 2 are two extreme examples. The initial driver end width is $1.9 \mu \mathrm{m}$. Therefore the distance between two adjacent 


\begin{tabular}{||c|c|c|c|c|c||c|c||}
\hline Case & $\begin{array}{c}\text { Driver end } \\
\text { width }(\mu m)\end{array}$ & $\begin{array}{c}\text { Load end } \\
\text { width }(\mu m)\end{array}$ & $\begin{array}{c}\text { Number of } \\
\text { iterations }\end{array}$ & $\log (c)$ & Delay(ns) & $\begin{array}{c}\log (c) \\
\text { Search }\end{array}$ & $\begin{array}{c}\text { Delay(ns) } \\
\text { Search }\end{array}$ \\
\hline 1 & 1.5207 & 0.7692 & 3 & -12.9368 & 0.4415 & -12.9365 & 0.4415 \\
\hline 2 & 1.6239 & 0.8392 & 2 & -13.0148 & 0.3970 & -13.0130 & 0.3970 \\
\hline 3 & 1.9284 & 0.9438 & 2 & -13.0958 & 0.3594 & -13.0944 & 0.3594 \\
\hline 4 & 7.4551 & 2.0168 & 4 & -13.4827 & 0.09181 & -13.4727 & 0.09178 \\
\hline
\end{tabular}

Table 2. Calculated optimal shapes and delays for case 1 to 4

\begin{tabular}{||c|c|c|c|c||}
\hline Case & $R_{d}(\Omega)$ & $c_{c}(f F)$ & $D(\mu m)$ & $w_{0}(\mu m)$ \\
\hline 1 & 100 & 0.4 & 3 & 1.9144 \\
\hline 2 & 100 & 0.2 & 3 & 1.9144 \\
\hline 3 & 100 & 0.2 & 10 & 1.9144 \\
\hline 4 & 10 & 0.2 & 10 & 8.0993 \\
\hline
\end{tabular}

Table 1. Circuit parameters and initial driver end width $w_{0}$.

wires is only about $1 \mu \mathrm{m}$. Case 4 is another similar extreme example, where the driver end width is $8 \mu \mathrm{m}$, so the distance between two adjacent wires is $2 \mu \mathrm{m}$. We expect that in these three cases the coupling capacitance plays an important role in determining the optimal shape. Case 3 is an opposite extreme example, where the distance between two adjacent wires is about $8 \mu \mathrm{m}$. Therefore in case 3, coupling capacitance may not play an important role in determining the optimal shape. These extreme examples are chosen to test the efficiency of our algorithm.

The first result we want to show is that in all cases we have studied, the delay $T_{D}$ is convex in the driver end width $w_{0}$ (also in $c$ ). The calculation is done by choosing a range of $w_{0}$ (thus we get a range of $c$ ) for each case. The delay can be calculated within this range. In Fig.4, $T_{D}$ is plotted versus $c$ in all cases. It is clear that $T_{D}$ is convex in $c$. Equation (3) shows that $c$ is also convex in $w_{0}$, therefore $T_{D}$ is convex in $w_{0}$.
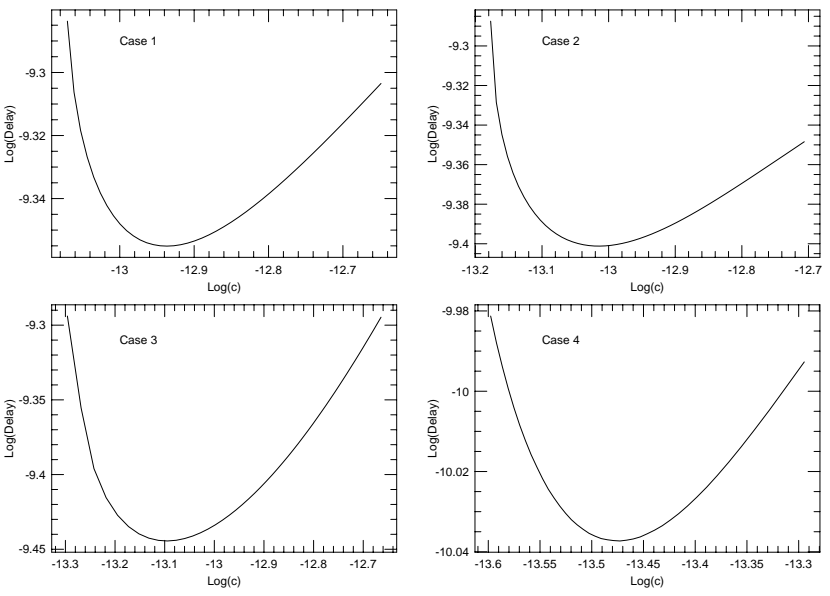

Figure 4. Numerically calculated delay is shown as a convex function of $c$ in four case studies.

The results of finding the optimal shape function are shown in Table 2 and Fig.5. In Table 2, we list the optimal solutions by using our algorithm in first six columns. The optimal solutions are listed as the driver end width and load end width, the number of iterations to get the optimal solutions, the optimal value of $c$ in logarithm and the calculated delay for each case. Last two columns are the optimal $c$ 's and delays found using binary search (labeled with "search" in the table). Comparing the last four columns, we find that the value of $c$ found by our algorithm is indeed the optimal value to minimize $T_{D}$. But our algorithm converges within only several iterations. In a SUN Sparc Station 5, it takes about 0.2 seconds for one iteration in all cases with a relative error of $1.0 \times 10^{-6}$. This indicates that our algorithm also works very fast. The calculated optimal shapes are plotted in Fig.5, where solid lines represent optimal shapes considering coupling capaci- tance and dashed lines represent optimal shapes without considering coupling capacitance. To our expectation, the optimal shapes in case 1 and 2 differ much from the optimal solutions without considering coupling capacitance. Because of the coupling capacitance, the wire should be made thinner in order to minimize its coupling effect. In case 4 , it does not differ that much because two adjacent wires are not close enough. In case 3, the two shapes are almost the same just because the coupling capacitance is not comparable to the area or fringing capacitance.
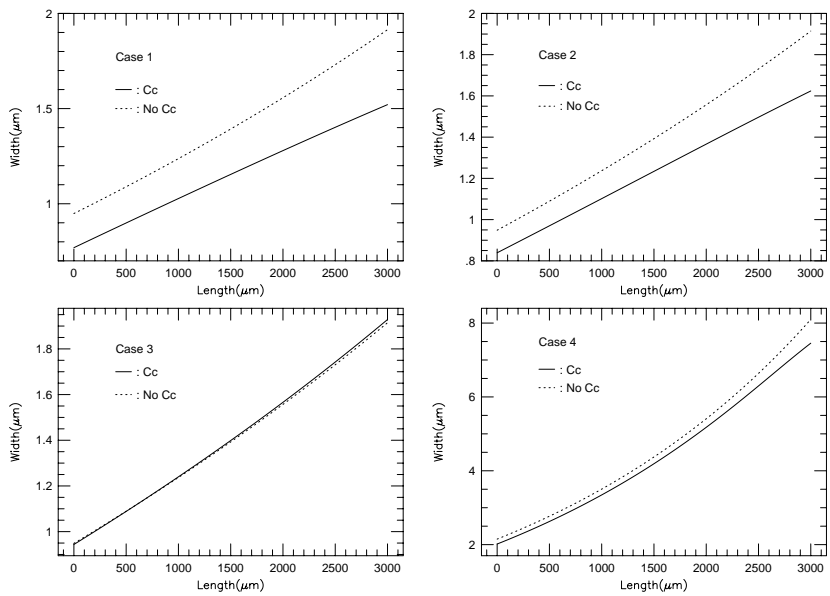

Figure 5. Numerically calculated optimal shapes.

References

[1] C.-P. Chen, Y.P. Chen, and D.F. Wong, Optimal wire-sizing formula under the Elmore delay model, Proc. ACM/IEEE DAC, pp.487-490, 1996.

[2] C.-P. Chen and D.F. Wong, Optimal wire-sizing function with fringing capacitance consideration, Proc. ACM/IEEE DAC, pp.604-607, 1997.

[3] R.M. Corless, G.M. Gonnet, D.E.G. Hare, D.J. Jeffrey and D.E. Knuth, On the Lambert W function, Tech. Report CS-93-03, Department of Computer Science, University of Waterloo, Canada.

[4] W.C. Elmore, The transient response of damped linear network with particular regard to wide-band amplifier, Journal of Applied Physics, vol.19, pp.55-63, 1948

[5] L.E. Elsgolc, Calculus of Variations, Pergamon Press Ltd., 1962.

[6] J.P. Fishburn, Shaping a VLSI wire to minimize Elmore delay, Proc. ED\&TC, 1997.

[7] J.P. Fishburn and C.A. Schevon, Shaping a distributed RC line to minimize Elmore delay, IEEE Transactions on CAS-I, vol.42, pp.1020-1022, 1995.

[8] Y. Gao and D.F. Wong, Optimal shape function for a bi-directional wire under Elmore delay model, ICCAD, pp.622-627, 1997.

[9] J. Rubinstein, P. Penfield, and M.A. Horowitz, Signal delay in RC tree network, IEEE Transaction on CAD, vol.CAD-2, pp.202-211, 1983

[10] T. Sakurai and K. Tamaru, Simple formulas for two- and three-dimensional capacitance, IEEE Transactions on ED, vol.ED-30, No.2, pp.183-185, 1983.

[11] T. Sakurai, Closed-form expressions for interconnection delay, coupling, and crosstalk in VLSI's, IEEE Transactions on ED, vol.ED-40, No.1, pp.118-124, 1993.

[12] S. Thakur, K.-Y. Chao and D.F. Wong, An optimal layer assignment algorithm for minimizing crosstalk for three VHV channel routing, Proc. IEEE ISCS, 1995

[13] Hai Zhou and D.F. Wong, An optimal algorithm for river routing with crosstalk constraints, ICCAD, pp.310, 1996 